\title{
On-line Group Decision Support by HIPRE 3+ Group Link
}

\author{
Raimo P. Hämäläinen and Eero Kettunen \\ Systems Analysis Laboratory. Helsinki University of Technology \\ Otakaari $1 M$, FIN-02150 Espoo, Finland \\ Tel. $(3580)+513054$, Telefox (358 0) 4513096 \\ e-mail: raimo@hut.fi
}

\begin{abstract}
HIPRE $3+$ Group Link is a decision support software for group decision making. The software is based on the interval AHP also known as the preference programming technique. HIPRE 3+Group Link can be used in group meetings to support the decision making process where individual AHP models are combined into a preference programming model. HIPRE $3+$ Group Link provides an interactive platform for the on-line analysis and combination of preferences in a group. This paper illustrates how to carry out a group decision process by using the preference programming technique.
\end{abstract}

Keywords: AHP, decision theory, GDSS, preference programming

\section{Introduction}

The preference programming technique allows imprecise preference statements in the analytic hierarchy process (AHP) $[2,6,8,10,11,13,14]$. In preference programming each pairwise preference ratio is given as an interval of numbers instead of a single number estimate (see Figure 1). The new technique with intervals of ratio judgments is especially suitable for group decision support. The intervals can be defined so that they include all preferences within the group. This is a totally new multicriteria approach to support group decision processes.

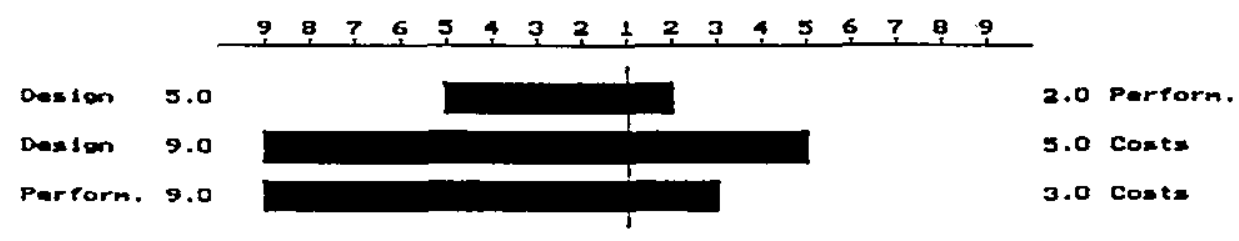

Figure 1: Pairwise interval preference statements. 
The idea of group decision support by the preference programming technique was first proposed by Hämäläinen et. al. in 1991 [7]. This first study tested the method in a real energy policy problem. The experiences of preference programming in group decision support are promising. The reader is encouraged to consult the references to learn more about the theoretical foundations of the preference programming approach $[6,9,10,12,13,14]$.

HIPRE 3+ Group Link (Group Link) [4] is a group decision support sottware that applies the preference programming technique. Group Link provides an interactive platform for the on-line analysis and combination of preferences in a group. In the online analysis individual AHP models are combined into a preference programming model through a local area PC-network. The software can be used in group meetings to support the decision making process, or in any research assignment where several AHP models are to be combined into a preference programming model.

To use the group link features of Group Link, you need a full copy of the HIPRE 3+software [5]. HIPRE $3+$ is needed to elicit the priorities of the individual group members. However, you also can do group analysis based only on the preference programming method without HIPRE $3+$. Then you are, in fact, nunning our INPRE interval AHP software.

The following chapters will give you an easy introduction to group decision support by the preference programming technique. As a demonstration problem we have a family selecting a new car. There are a number of different approaches you can take to the group analysis by the interval technique. Here we only describe the principles but not all the possible ways to carry out the group decision process.

\section{Family's car selection problem}

This example deals with a family which is going to buy a new car. The family consists of four members: Father, Mother, Daughter and Son. The family has already reduced the number of alternatives to three cars, namely the top models of $B M W$, Cadillac and Hondz. They have also decided to use Group Link to support their group decision problem.

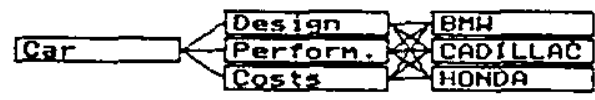

Figure 2: Decision hierarchy for the family's car sclection problem.

The criteria are 'Design', 'Performance' and 'Costs' (see Figure 2). 'Design' covers factors like style, safety and comfort. 'Performance' represents both the speed and the acceleration of the car. The 'Costs' criterion includes both price of the car and its maintenance costs. 


\subsection{Individual prioritizations}

Father is the first one to assess his preferences. To him the costs are five times more important than design and three times more important than performance as he is the one who has to pay for the car. Father's pairwise comparison matrices are:
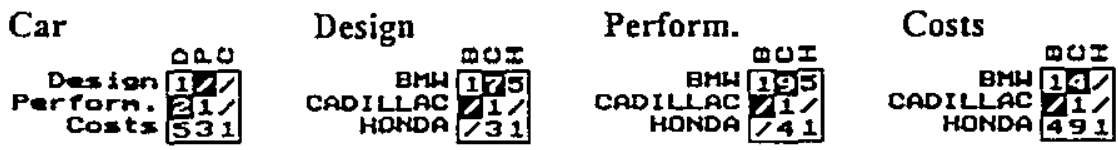

Figure 3: The comparisons given by Father.

Mother doesn't care about money. She likes comforrable cars - design is the most important thing in her opinion. Mother's pairwise comparison matrices are:
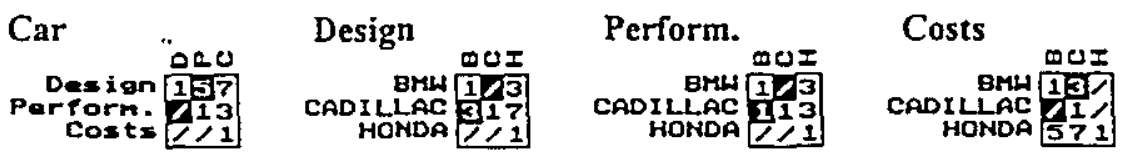

Figure 4 : The comparisons given by Mother.

The teenage Daughter is fond of fast and beautiful cars. She also thinks that money is unimportant. Daughter's pairwise comparison matrices are:
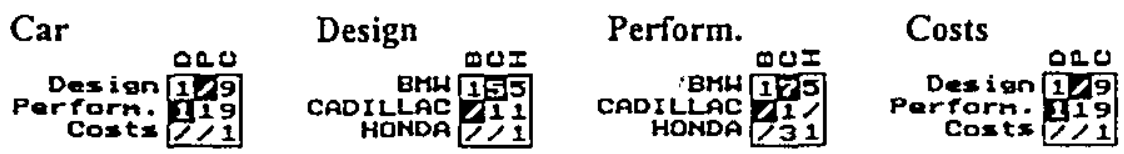

Figure 5: The comparisons given by Daughter.

The fourteen year old Son finds design, performance and costs equally important. Son's pairwise comparison matrices are:
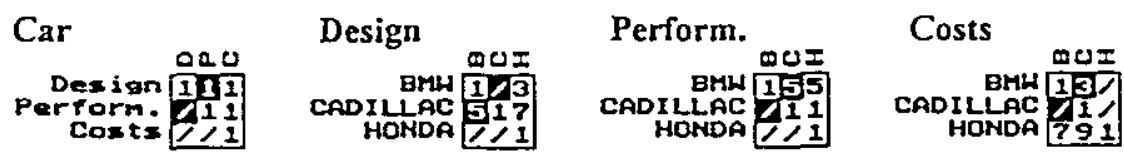

Figure 6: The comparisons given by Son.

The resulting composite priorities for each family member are shown in Figure 7. 

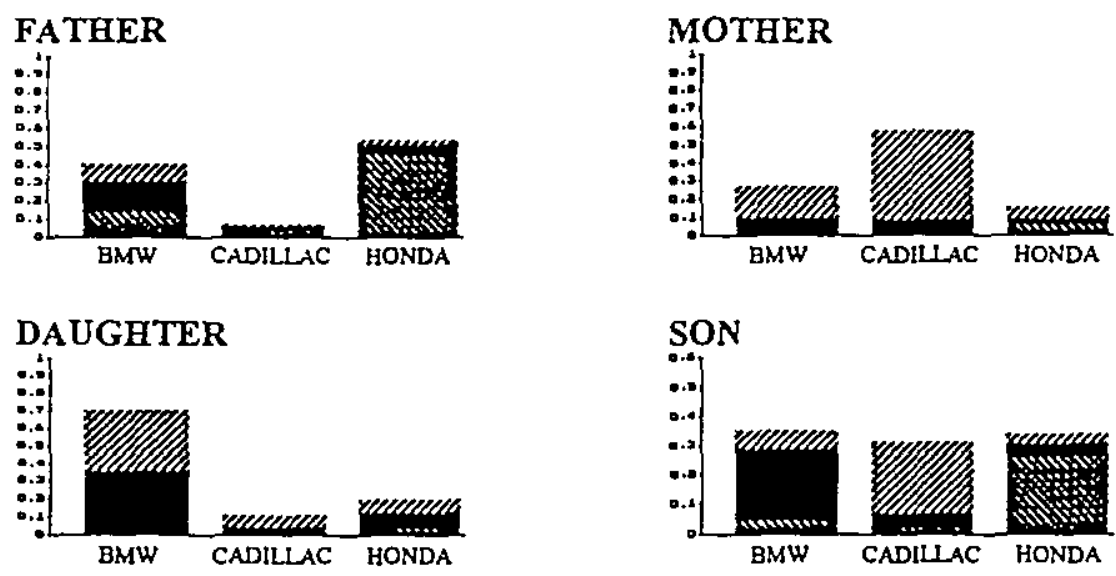

Wh Design
Performance
Costs

Figure 7: Individual composite priorities as shown by HIPRE $3+$.

2.2. Analysis of the group decision with HIPRE 3+ Group Link

\begin{tabular}{|l|ll|ll|}
\hline FAMILY.GRP & PAEU & Next & ESC & SAlect \\
\hline FATHER.MOD & & & \\
MOTKER.MOD & & \\
DAUGHTER.MOD & & & \\
SON.MOD Af & & & \\
& & &
\end{tabular}

Figure 8: Group definition.

With Group Link you select the members whose prioritizations you want to include in the preference programming model. In this case Father, Mother, Daughter and Son are selected to be members of a group 'family' (see Figure 8). Group Link combines the selected individual prioritizations into a group preference programming model.

The weight intervals for the alternatives are shown in the top right hand corner of the screen (Figure 9). The bars reflect the differing opinions of the family. The numbers (the so called ambiguity indexes) in each element of the hierarchy indicate how wide the local priority intervals are. This gives you a measure of consensus on the criteria. and alternative weights. If the value is close to zero, the group's judgments under that criterion are similar. A value close to one indicates that there are essential differences in the preferences. 


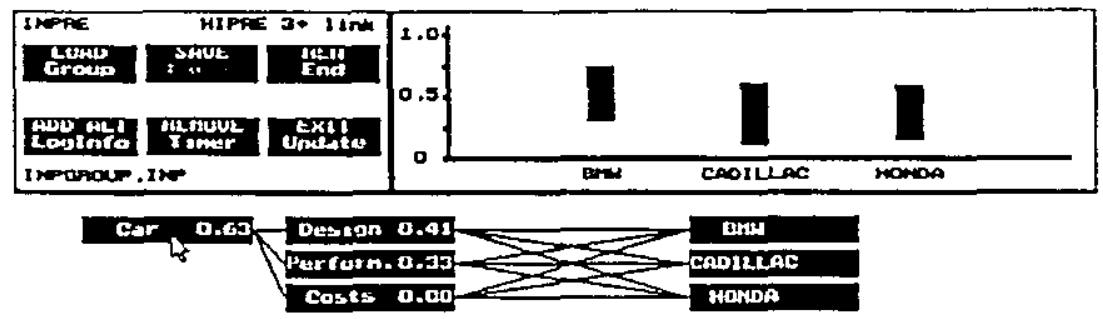

Figure 9: The main screen of the preference programming analysis.

It can be seen that none of the three cars is considered to be clearly better than the others, i.e. no car dominates any other car. The wide intervals in all of the elements except 'Costs' indicate that the family has strong differences in the preferences about the performance of the alternatives on these criteria. Often it is advisable to begin the group process by discussing the criteria that have the highest ambiguity index. In this case the top element 'Car' has the value 0.63 , which means that there are strong differences in the preferences about the relative imporance of the main criteria design, performance and costs.

Father was the only one to say that 'Costs' is more important than 'Design' and 'Performance', and 'Performance' is more important than 'Design'. Thus Father's judgments represent the right hand end of each interval. Daughter thought that both 'Design' and 'Performance' are nine times more important than 'Costs'. These judgments are in the left hand end of the last two intervals. Mother's opinion that 'Design' is five times more important than 'Performance' defines the left hand end of the first interval in Figure 10.

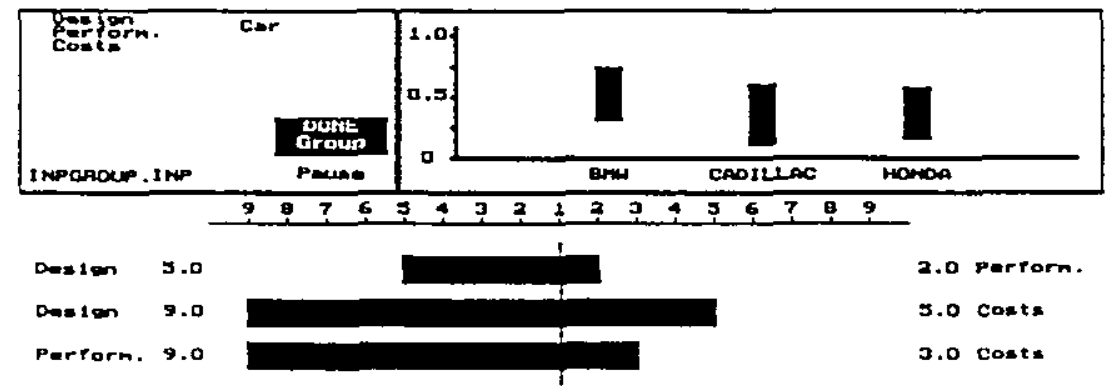

Figure 10: The preference intervals under the criterion 'Car'.

Let us imagine that the family has recentiy won in a lottery. Thus Mother can convince Father that money is not as important as he thinks. After some time and support from Son's and Daughter's side Father decides to change his opinion on 'Costs' (see Figure 11). The gray parts of the intervals represent the range of inconsistent statements. 
Inconsistent statements are not taken into account in the analysis. The dark parts of the bars show the active bounds on the feasible weights.

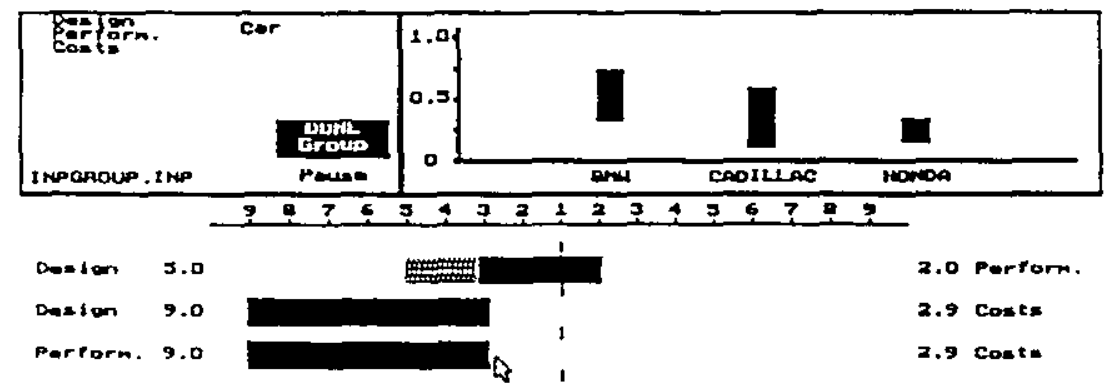

Figure 11. The preference intcrvals after the change under the criterion 'Ca'r'.

Now the family cannot get any further in reducing the differences of opinion under this criterion, so they leave the preferences under the criterion 'Car' as such and move on to the next criterion.

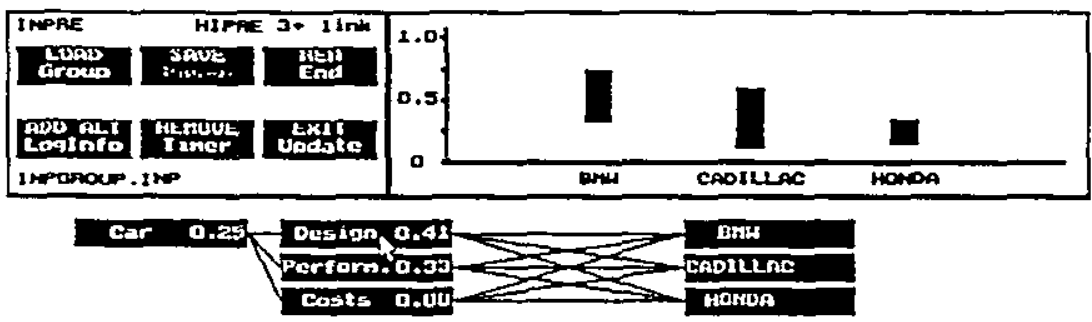

Figure 12: The main screen after the change.

The highest value for the ambiguity index is now 0.41 for the element 'Design' (see Figure 12). This is the criterion with the widest disagreement between the members of the family and it is discussed next. 


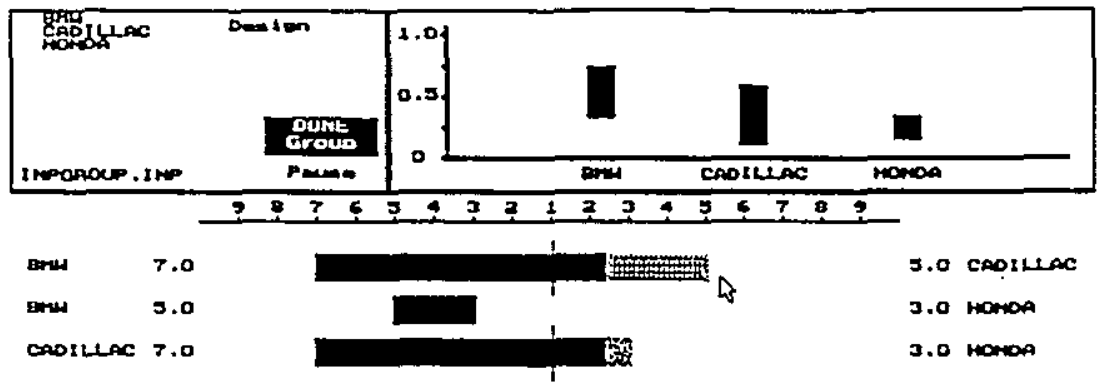

Figure 13: The preference intervals under the criterion 'Design'.

Mother and Son like Cadillac's design more than BMW's. Father and Daughter disagree with them. After a short discussion, Father and Daughter can convince Mother and Son that BMW is of better design than Cadillac. They agree to change the right hand end of the first interval so that BMW is at least five but not more than seven times better than Cadillac (see Figures 13 and 14).

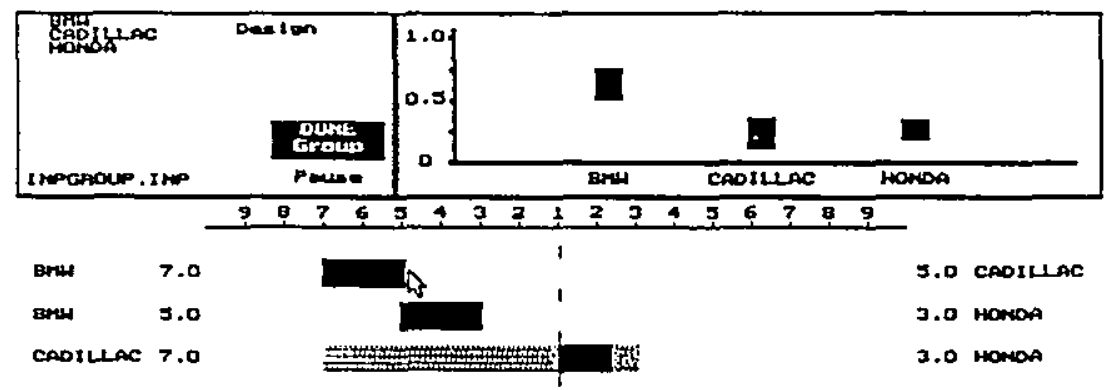

Figure 14: The revised preference intervals under the criterion 'Design'.

After this revision BMW dominates both Cadillac and Honda. This can be seen from the weight intervals in the top right comer of the screen in Figure 14. BMW thus becomes the family's choice. You may want to go back to analyze the local prioritizations. The remaining intervals under each element represent the preference statements which would result in BMW being the best alternative.

\subsection{Subgroup analysis}

Sometimes it is useful to do analysis with subgroups. In the family's case you can define subgroups as follows: group 'girls' contains Mother and Daughter, group 'boys' contains Father and Son. You might also be interested in analyzing the subgroups 'adults' and 'children'.

By subgroup analysis you may want to find members with the same kind of preferences and possibly suggest solutions based on the choice of a majority coalition. In the 
family's case the girls seem to prefer Honda less than the boys do. This can be seen by comparing the weight intervals of the alternatives in Figure 15. There are differing preferences between the two groups under the main criterion 'Car' (see Figure 16). For example, the girls prefer design from seven to nine times more than costs. The boys prefer costs up to five times more than design.

You may want to change the individual AHP models with HIPRE 3+ during the group analysis phase. With Microsoft Windows you can run one or more HIPRE $3+$ programs and Group Link simultaneously as separate MS-DOS processes. This is done by opening two or more full size MS-DOS windows. In one of the windows you run Group Link and in the others you run HIPRE 3+ (to move between these windows press $[\mathrm{Alt}]+[\mathrm{TAB}])$. This arrangement allows you to change the prioritizations in the AHP models with HIPRE $3-$ and then update the group model with Group Link.
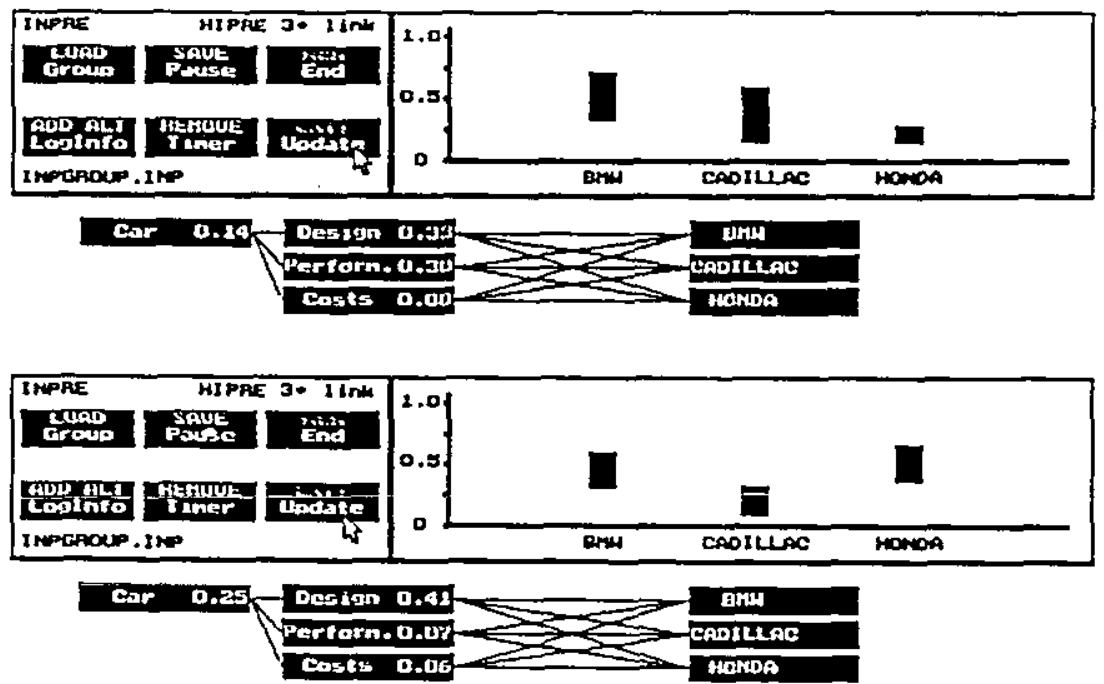

Figure 15: The preference programming models of the group 'girls' and the group 'boys'. 


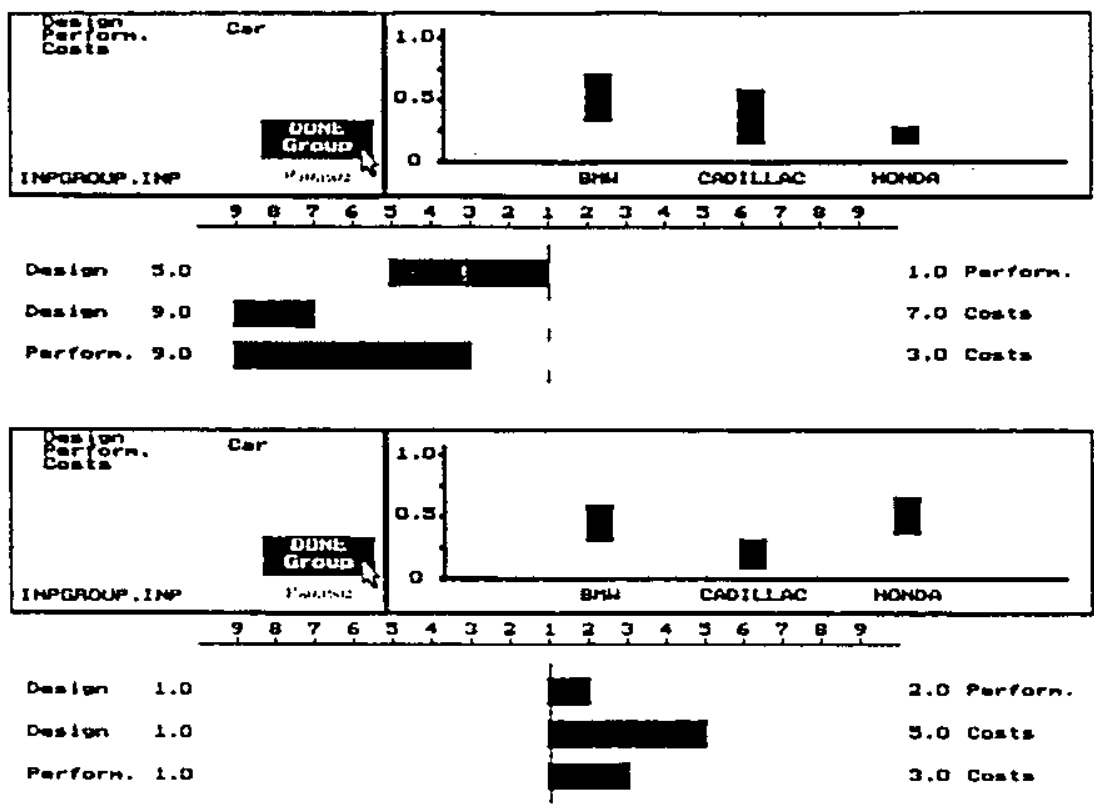

Figure 16: The interval preferences of the group 'girls' and the group 'boys' under the element 'Car'.

\section{On-line group decision support}

In the previous chapter the decision process was carried through in a single computer. In this chapter we illustrate the group process in a local area PC-network environment.

When a local area PC-network is available, Group Link can be used to support a real time group meeting. In this case the group members make their individual prionitizations with personal workstations running HIPRE 3+. Group Link can be run in one dedicated workstation or in the decision makers' workstations under Microsoft windows. In the dedicated workstation Group Link combines the group's preferences on-line. The resulting preference programming model can be shown on a general screen so that all the group members can follow the development of the group priorities. If the Microsoft Windows is available, the decision maker run both HIPRE $3+$ and Group Link simultaneously and thus can follow the decision process with his own computer. These techniques make several different kinds of negotiation procedures possible.

First the group members enter their individual preferences with HIPRE $3+$. The individual prioritizations are combined with Group Link into a group preference programming model. The group then discuss the disagreements shown by the intervals in the model and changes the judgments in the group model. This is similar to what the family did in this paper.

Another way is that the negotiators change their prioritizations in their individual AHP models instead of changing the interval judgments in the group model. The group 
model is updated at a given time interval and the disagreements are discussed. The group members again aim to change their individual prioritizations so that the group intervals will narrow. In this mode the decision makers always have their own priorities available. It is an interesting topic for future research to evaluate the differences between the available approaches. The role of the facilitator and other situational issues such as face to face or remote meetings are also problems which can be approached by this software.

A simple way is to start directly with the group's preference programming model. In this case no individual AFP prioritizations are made.

The main idea in all the procedures is that the group can focus the discussion on the prioritizations which have the highest differences in opinion. The disagreements can be seen in the group model by the ambiguity indexes.

\section{Conclusion}

In this paper we have introduced an approach to group decision support by the HIPRE $3+$ Group Link software. The preference programming technique used by the software is a new promising method to support group decision making. The preference intervals guide the discussion towards the prioritizations which have the most conflicting views. In group meetings this is likely to be an efficient way to find the dominating alternative. Often it is possible to find this afternative even under relatively wide differences in opinion. Then the group does not have to agree upon everything and the need to force the group to unanimity about the importance of criteria can be avoided. This again is a situation which definitely increases the user acceptance of the method.

When the preference programming technique has been tested in group meetings the participants have found the technique a useful tool in reaching a consensus. The interactiveness of the approach has been seen as an essential advantage. HIPRE $3+$ Group Link is available from us to all interested researchers to make it easier to gain further experiences of preference programming in group decision support. 


\section{References}

1 Arbel, A., Approximate articulation of preference and priority derivation European Joumal of Operational Research 43, 317-326 (1989).

2. Arbel, A. and Vargas, L.G., Preference simulation and preference programming: robustness issues in priority derivation. European Joumal of Operational Research 69, 200-209 (1993).

3. Dyer, R.F. and Forman, E.H., Group decision support with the analytic hierarchy process. Decision Support Systems 8, 99-124 (1992).

4. Hämäläinen, R.P and Kettunen, E., HIPRE 3+Group Link User's Guide. Helsinki University of Technology, Systems Analysis Laboratory (1994).

5 Hämäläinen, R.P and Laur, H., HIPRE 3+User's Guide. Helsinki University of Technology, Systems Analysis Laboratory (1992).

6. Hämäläinen, R.P. and Pöyhönen M., On-line group decision support by preference programming in traffic planning. Mamuscript (1994).

7. Hämäläinen, R.P., Salo, A.A. and Pöysti K., Observations about consensus seeking in a multiple criteria environment. Proceedings of the Twenty-Fifth Hawaii International Conference on System Sciences, Vol. IV, IEEE Computer Society Press Los Alamitos, California, 190-198 (1991).

8. Saaty, T.L., The Analytic Hierarchy Process. McGraw-Hill, New York (1980).

9. Salo, A.A., Inconsistency analysis by approximately specified priorities. Mathematical and Computer Modeling 17, 4-5, 123-133 (1993).

10. Salo, A.A. and Hämäläinen, R.P., Decision support under ambiguous preference statements. Proceedings of the AIRO'OO Ammal Conference of the Italian Operational Research Society, Sorrento, Italy, 229-243 (1990).

11. Salo, A.A. and Hämäläinen R.P., Interactive decision support through interval judgments. Pruceedings of the Second International Symposium on the Analytic Hierarchy Process, Pittsburg, U.S.A., 215-226 (1991).

12. Salo, A.A. and Hämäläinen, R.P., Preference assessment by imprecise ratio statements. Operations Research 40, 6, 1053-1061 (1992).

13. Salo, A.A. and Hämäläinen, R.P., Processing interval judgments in the analytic hierarchy process. Multiple Criteria Decision Making, A. Goicoechea, L. Duckstein, S. Zionts (eds.), Springer-Verlag, New York, 359-371 (1992).

14. Salo, A.A. and Hämäläinen, R.P., Preference programming through approximate ratio comparisons. Forthcoming in European Journal of Operational Research. 